\title{
Portable fluorescence lifetime spectroscopy system for in-situ interrogation of biological tissues
}

Marcelo Saito Nogueira

Alessandro Cosci

Ramon Gabriel Teixeira Rosa

Ana Gabriela Salvio

Sebastião Pratavieira

Cristina Kurachi 


\title{
Portable fluorescence lifetime spectroscopy system for in-situ interrogation of biological tissues
}

\author{
Marcelo Saito Nogueira, ${ }^{a}$ Alessandro Cosci, ${ }^{a}$ Ramon Gabriel Teixeira Rosa, ${ }^{a}$ Ana Gabriela Salvio, \\ Sebastião Pratavieira, ${ }^{a, *}$ and Cristina Kurachi ${ }^{\mathrm{a}}$ \\ aUniversity of São Paulo, São Carlos Institute of Physics, São Carlos, São Paulo, Brazil \\ ${ }^{\mathrm{b}}$ Amaral Carvalho Hospital, Jahu, São Paulo, Brazil
}

\begin{abstract}
Fluorescence spectroscopy and lifetime techniques are potential methods for optical diagnosis and characterization of biological tissues with an in-situ, fast, and noninvasive interrogation. Several diseases may be diagnosed due to differences in the fluorescence spectra of targeted fluorophores, when, these spectra are similar, considering steady-state fluorescence, others may be detected by monitoring their fluorescence lifetime. Despite this complementarity, most of the current fluorescence lifetime systems are not robust and portable, and not being feasible for clinical applications. We describe the assembly of a fluorescence lifetime spectroscopy system in a suitcase, its characterization, and validation with clinical measurements of skin lesions. The assembled system is all encased and robust, maintaining its mechanical, electrical, and optical stability during transportation, and is feasible for clinical measurements. The instrument response function measured was about $300 \mathrm{ps}$, and the system is properly calibrated. At the clinical study, the system showed to be reliable, and the achieved spectroscopy results support its potential use as an auxiliary tool for skin diagnostics. $\odot 2017$ Society of Photo-Optical Instrumentation Engineers (SPIE) [DOI: 10.1117/1.JBO.22.12.121608]
\end{abstract}

Keywords: portable; fluorescence lifetime; fluorescence spectroscopy; biological tissue; optical diagnosis.

Paper 170352SSR received May 31, 2017; accepted for publication Sep. 22, 2017; published online Oct. 19, 2017.

\section{Introduction}

Steady-state fluorescence spectroscopy and time-resolved fluorescence spectroscopy of biological tissues have been presented as techniques of great potential for tissue characterization with diagnostic purposes. ${ }^{1-5}$ This potential is due to the main advantages of optical technologies, which include the possibility of evaluating the tissue metabolism in situ, without the removal and processing of the biological sample, through a fast and noninvasive procedure. ${ }^{6-9}$ Among these techniques, steady-state fluorescence spectroscopy has been widely used to extract biological and biochemical information related to the molecular composition of tissues, which is complementary to the information associated to the fluorophores microenvironment obtained from fluorescence lifetime (time-resolved) spectroscopy. ${ }^{10,11}$ When these techniques are combined with other tools, they also allow the investigation of fluorescence quenching, Stokes shift, polarization, anisotropy, fluorescence resonance energy transfer, and fluorescence correlation spectroscopy. ${ }^{11}$

For fluorescence lifetime measurements, there are two modalities: time-domain fluorescence lifetime (TDFL) and frequency-domain techniques. The first one is based on the temporal distribution of photon arrival times after each excitation pulse, ${ }^{12}$ while the second one relies on the phase shift or amplitude modulation as a function of the frequency of modulated excitation that strikes the sample. ${ }^{11}$ One of the most used TDFL techniques is the time-correlated single photon counting (TCSPC), which is the technique used in this study. The principle of TCSPC is to measure the time between the pulse of light and the photon that arrives at the detector. Using a high

*Address all correspondence to: Sebastião Pratavieira, E-mail: prata @ifsc.usp .br repetition rate in a brief period $(\sim 1 \mathrm{~ms})$, it is possible to count a statistical number of photons that allow the construction of a histogram graph of the number of detected photons as a function of time. For fluorescence, this histogram represents the convolution of the detector response and the fluorescence decay over time. For biological molecules, a typical decay time is usually at the nanosecond scale. ${ }^{10,11}$ In TCSPC technique, real decay times are measured only when one photon is recorded per detection period. On the other hand, measurements of apparently shorter lifetimes occur when more than one photon is detected in the same period. This is called pileup effect. To avoid photon pileup, the excitation rate should be much lower than the laser pulse repetition rate. This typically means that the detection rate, which limits the number of acquired photons in the time range of each measurement, must be below $5 \%$ of the pulse repetition rate. ${ }^{11,13}$ This condition is highly necessary to obtain a better uniformity of measurements for each sample, especially for clinical systems, since targeted biological tissues are complex media, considering the interrogation of their optical process and biochemical changes.

Biological tissue autofluorescence arises primarily from specific endogenous fluorophores located in mitochondria (nicotinamide adenine dinucleotide phosphatase, flavin coenzymes, porphyrins, and lipopigments), structural proteins in extracellular matrix (collagen, elastin, and keratin), cell lysosomes (lipofuscins), and several aromatic amino acids appearing in many proteins within the living body. ${ }^{11}$ These fluorophores are monitored when biochemical alterations, such as variations in the number of collagen cross-links, elastin levels, keratinization, pigmentation, and selective accumulation of porphyrin derivatives, are

1083-3668/2017/\$25.00 @ 2017 SPIE 
present, thus allowing disease detection with all the advantages of optical techniques. One imaging technique to optically differentiate between most of these diseases is to choose to target fluorophores with different fluorescence spectra. However, when these fluorophores have similar fluorescence spectra, such as the ones for free and bound nicotinamide adenine dinucleotide (NADH), tissue changes may be observed with fluorescence lifetime techniques. For these reasons, tissue discrimination may be achieved using noninvasive procedures, such as fluorescence lifetime measurements, since they may provide information about the metabolism of the interrogated tissues by targeting NADH, flavin adenine dinucleotide (FAD), and biomolecules involved in cellular respiration. ${ }^{5,14-16}$

Clinical fluorescence lifetime systems described in the literature are divided into imaging systems (laser scanning) or single-point spectroscopy measurements. Most of the reported imaging systems can perform multimodal optical measurements, ${ }^{17-27}$ but their portability is still a challenge, mostly due to their size. This size limitation makes clinical measurements possible only at the place where the system is located. Moreover, to have a sufficient statistics of photons, measurements may require a long acquisition time $(>10 \mathrm{~s}) .{ }^{28}$ On the other hand, systems for single-point measurements offer more possibilities for clinical investigation in several places as a consequence of their relatively small size and portability. The use of fiber optic probes also makes them more flexible and versatile and provides the possibility to couple the system to commercial endoscopes. All these features make single-point measurement systems ideal to be used for fluorescence lifetime evaluation in the clinical environment, providing the option of a system that is more user-friendly and easier to be transported compared to current developed systems. Clinical in-vivo fluorescence lifetime measurements using portable systems are relevant, since most of these measurements are performed in cell cultures, and ex-vivo normal and abnormal tissues. Recently, various single-point systems ${ }^{29-32}$ were reported for ex-vivo cancer detection or in-vivo measurements of skin and gastrointestinal lesions.

Most studies using fluorescence lifetime techniques focus on the discrimination among skin lesions using a small number of skin lesions. One of the first studies is from De Beule et al., ${ }^{33}$ who reported a significant difference when comparing six basal cell carcinomas (BCCs) with surrounding uninvolved skin at 435-nm excitation, although normal tissue and BCCs could not be discriminated using $355 \mathrm{~nm}$. Later, Galletly et al. ${ }^{34}$ were able to distinguish 25 areas of $\mathrm{BCC}$ from surrounding skin in an $e x$ vivo study with the same laser excitation $(355 \mathrm{~nm}), 375-$ and 455-nm longpass filters.

A comparison among skin lesions was performed by Patalay et al., ${ }^{35}$ who showed a significant statistical difference between four benign naevi and three nodular BCCs using 760-nm excitation and detection channels higher or lower than $500 \mathrm{~nm}$. Also, using a 445-nm excitation, Thompson et al. obtained a very low $p$-value $\left(5.6 \times 10^{-6}\right.$, Wilcoxon signed-rank test $)$ when comparing the fluorescence lifetime from a set of lesions [BCC, squamous cell carcinoma (SCC), benign naevus, dysplastic naevus, malignant melanoma, and actinic keratosis (AK)] and normal skin. However, when comparing BCCs with normal tissue, this $p$-value increased for $0.002 .^{30}$ More investigation is needed on differences between fluorescence lifetimes of skin lesions, which makes the availability and accessibility of clinical systems appealing features for future research and analyses.
Current clinical systems are already able to perform multispectral and/or multiphoton fluorescence lifetime imaging and/or a set of second-harmonic generation, single- or multiphoton fluorescence, and optical tomography measurements. Potential applications for these systems include the diagnosis of BCCs, SCCs, AK, cutaneous keloids, and malignant melanomas (for skin); colonic polyps, inflammatory bowel disease, and diseased human laryngeal tissues (for endoscopy); and atherosclerotic lesions, brain tumors, and cervical intraepithelial neoplasia. ${ }^{17-27,34,36-43}$ However, most of them are set on an optical table or placed on a transportable breadboard. ${ }^{29-31}$ So far, only a few examples of portable and robust instrumentations for fluorescence lifetime spectroscopy are reported. ${ }^{44,45}$ These portable systems were designed for analysis of in-vitro samples $^{44}$ or chlorophyll analysis ${ }^{45}$ instead of clinical applications. They use LEDs as light sources, which have a relatively broad excitation band compared to lasers and, consequently, excite a higher number of fluorophores in biological tissues. This may hinder the identification of the source of fluorescence lifetimes in biomedical applications because of the broad overlap among both excitation and emission spectra of tissue fluorophores. ${ }^{11}$ Then, when the aim is to target very specific fluorophores, this approach may not be suitable.

In this study, we describe, to the best of our knowledge, the first portable single-point fluorescence lifetime systems capable of performing fluorescence spectroscopy and fluorescence lifetime measurements in clinics, its characterization, and its validation with measurements in biological tissues. Normal tissue, benign, and malignant skin lesions with clinically similar features were investigated as a primary clinical application.

\section{Materials and Methods}

\subsection{Fluorescence Lifetime Spectroscopy System}

The assembled time-resolved fluorescence system uses two lasers: one emitting at 378-nm wavelength and another at $445 \mathrm{~nm}$ (BDL-375-SMC and BDL-445-SMC, Becker and Hickl, Berlin, Germany). These wavelengths were primarily chosen to excite NADH and FAD molecules in biological tissues in vivo. The repetition rate can be set at 20,50 , or $80 \mathrm{MHz}$, and the laser pulse width is around 50 to $100 \mathrm{ps}$. The excitation light was coupled to a bifurcated fiber (BIF400-UV-VIS, Ocean Optics, Dunedin, Florida) with a common end of two $400-\mu \mathrm{m}-$ diameter fibers, positioned side-by-side. This optical fiber probe delivers the excitation light to the sample, and the collected light passes through bandpass filters before arriving at a hybrid photomultiplier tube (PMT) detector (HPM-100-50, Becker and Hickl, Berlin, Germany) with detection range from 400 to $900 \mathrm{~nm}$ and virtually free of after-pulsing effect, which could distort our fluorescence decay by generating delayed photon counts that are not expected in our measurements. These delayed counts may occur due to three processes: (a) when photoelectrons, produced by ionized atoms of the residual gas in photomultipliers, are accelerated toward the photocathode; (b) luminescence of this residual gas; (c) fluorescence effects of dynodes. $^{46,47}$ Two different bandpass filters at $440 \pm 20 \mathrm{~nm}$ and $514 \pm 15 \mathrm{~nm}$ were placed in front of the detector to mainly provide detection of NADH and FAD spectral emission regions at 378 and $445 \mathrm{~nm}$, respectively. In addition to these filters, longpass ones (405-nm longpass for 378-nm excitation and 475-nm longpass for 445-nm excitation) were used to measure the signal from a broader range of the emission spectrum. 


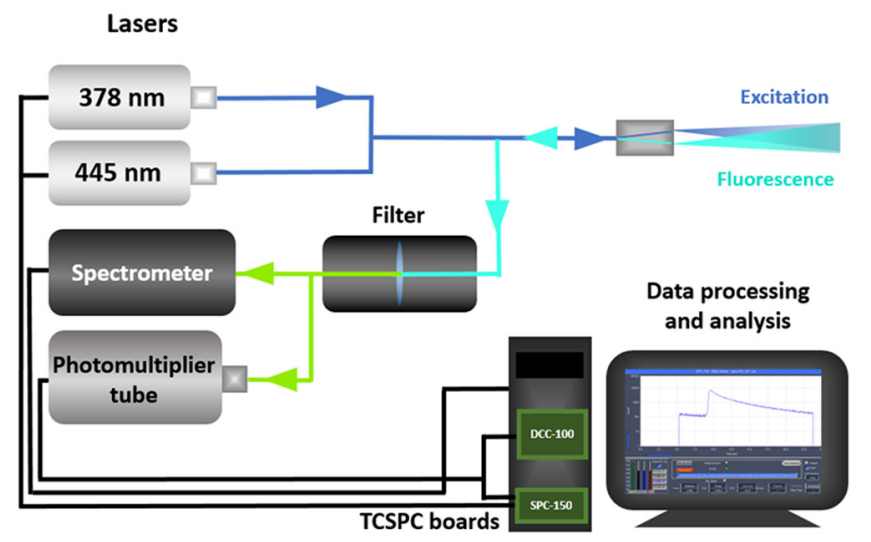

Fig. 1 Schematic drawing of the components for acquisition of fluorescence spectra and fluorescence lifetimes. The excitation light of one of the diode lasers (378 or $445 \mathrm{~nm}$ ) is delivered to the sample through a bifurcated fiber optic probe. The fluorescence and backscattered light of this sample are collected using the same probe, which will send the light to the filter holder. A combination of four possible filters can be used to remove the backscattered light and acquire the spectral region of interest: bandpass filters at $440 \pm 20 \mathrm{~nm}$ or at $514 \pm 15 \mathrm{~nm}$ and 405- or 475-nm longpass filters. The fluorescence goes to a spectrometer or a hybrid PMT, and, then, the fluorescence spectrum and fluorescence decay curve are measured. The PMT is connected to two TCSPC boards: the detector control module (DCC-100) and the time-correlated single photon counting module (SPC-150).

Fluorescence lifetime measurements were performed using TCSPC boards (SPC-150 and DCC-100, Becker and Hickl, Berlin, Germany). The same lasers (378 and $445 \mathrm{~nm}$ ) are used for fluorescence spectroscopy measurements. The fluorescence spectrum is collected using a portable spectrometer (USB2000-FLG, Dunedin, Florida). Figure 1 shows a scheme of the assembled experimental setup.

\subsection{Mechanical Encasement of the Clinical System}

A $48.5 \mathrm{~cm} \times 38.5 \mathrm{~cm} \times 19 \mathrm{~cm}$ metallic suitcase encases all the components, and the equipment can be plugged into an outlet using an IEC $60320 \mathrm{C} 13$ computer power cord. The mechanical structure of the system was planned so that it could be carried without relevant optical misalignments, unusual exposure to intense light sources, dust, and chemical substances. The front panel (consisting of the external controls of the lasers, filter exchange module, power button, and USB connections for mouse and keyboard) of the portable system becomes accessible to the user by support of a shelf in upper part inside the suitcase (Fig. 3), which also optimizes the arrangement for minimum volume and appropriate weight distribution. In addition, orientation and intercomponent distances were set to allow their fixation and suitable refrigeration.

Our group used this fluorescence lifetime spectroscopy system for successful in-vivo measurements. ${ }^{15,16}$ Previously, it was assembled over an optical bench and should be disassembled to be transported. However, after its assembly inside a suitcase, it can be carried without setting up cables and TCSPC boards connections in the place where the system will be used, transporting of separated parts, and assembling its structure. After transporting the suitcase by hand and/or in a vehicle, there were no significant differences in our calibration measurements (data not shown). When assembled in the suitcase, its volume was reduced to $38 \%$ (final volume: $38,166 \mathrm{~cm}^{3}$ ) of its previous

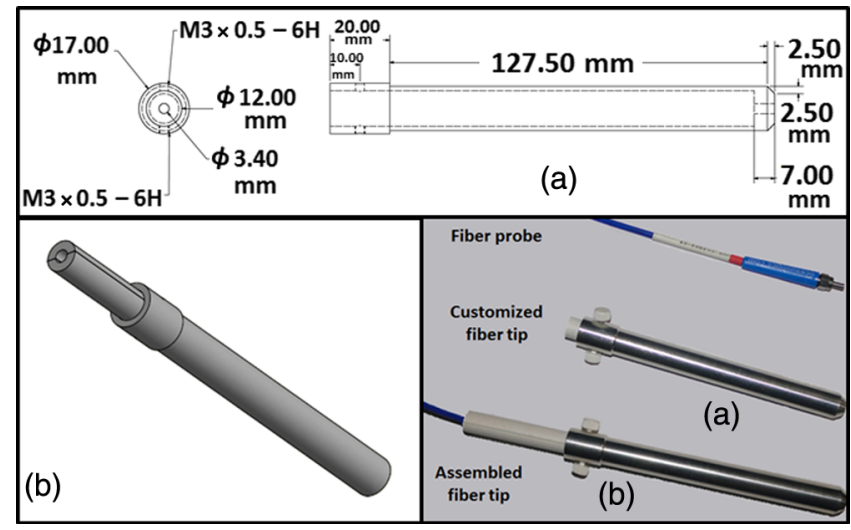

Fig. 2 Fiber tip used for more stable measurements in different environments. (a) Dimensions of fiber tip in its top view and cross-section. (b) Fiber tip assembled with the support structure (excluding screws) to hold the fiber optic probe inside the tip. A comparison of sizes of the fiber probe with and without the tip can be made in the photograph displaying each part of the assembled fiber tip.

one, as the original volume included the computer fixed in a separate larger structure. In addition to the decrease in system volume, it has mechanical, electrical, and optical stability and allows fast changing optical filters during clinical measurements. Furthermore, a metallic fiber tip (Fig. 2) was designed to provide easier handling by the system operator and, consequently, fewer variations of pressure, illumination, and angulation during clinical measurements. Since the optical system is optical fiber based, it is possible to reach any external part of the body for measurements, making this system available for direct or endoscopic measurements, and further coupling to fluorescence lifetime imaging microscopy measurements.

The system components are all enclosed in the suitcase, which includes a cover that eliminates contact between the system operator and electrical/optical components. This cover also provides a better-designed front panel, safety in clinical use, and protection in case of liquid or fluid spillage, as well as allows for surface disinfection. The control panel (Fig. 3) was customized to allow direct and immediate access to the most useful features for the system operator.

\subsection{System Characterization}

We performed measurements of the fluorescence lifetime of the fluorescent molecule Rhodamine $6 \mathrm{G}$ and the instrument response function (IRF) to evaluate the calibration of the system and determine the resolution of measured lifetimes. The temporal broadening of the laser pulse due to its propagation in multimode fibers, i.e., its temporal width, was measured after it passes through the excitation and collecting fibers and after it strikes a scattering medium [solution $0.5 \%$ Lipofundin ${ }^{\circledR}$ (Laboratories B. Braun S.A., Rio de Janeiro, Brazil) in distilled water]. A $2.5 \mu \mathrm{g} / \mathrm{mL}$ Rhodamine 6G (Sigma-Aldrich, St. Louis, Missouri) in distilled water solution, used for verifying the system calibration, was characterized by its absorption and fluorescence spectra using a 475-nm longpass filter, and 378 and $445 \mathrm{~nm}$ as excitation wavelengths. The average fluorescence lifetime of this solution was compared to the values reported in the literature for the assessment of the calibration.

To estimate the functionality of the customized fiber tip in the reproducibility of our in-vivo measurements, a preliminary test was performed by evaluating their standard deviations when 


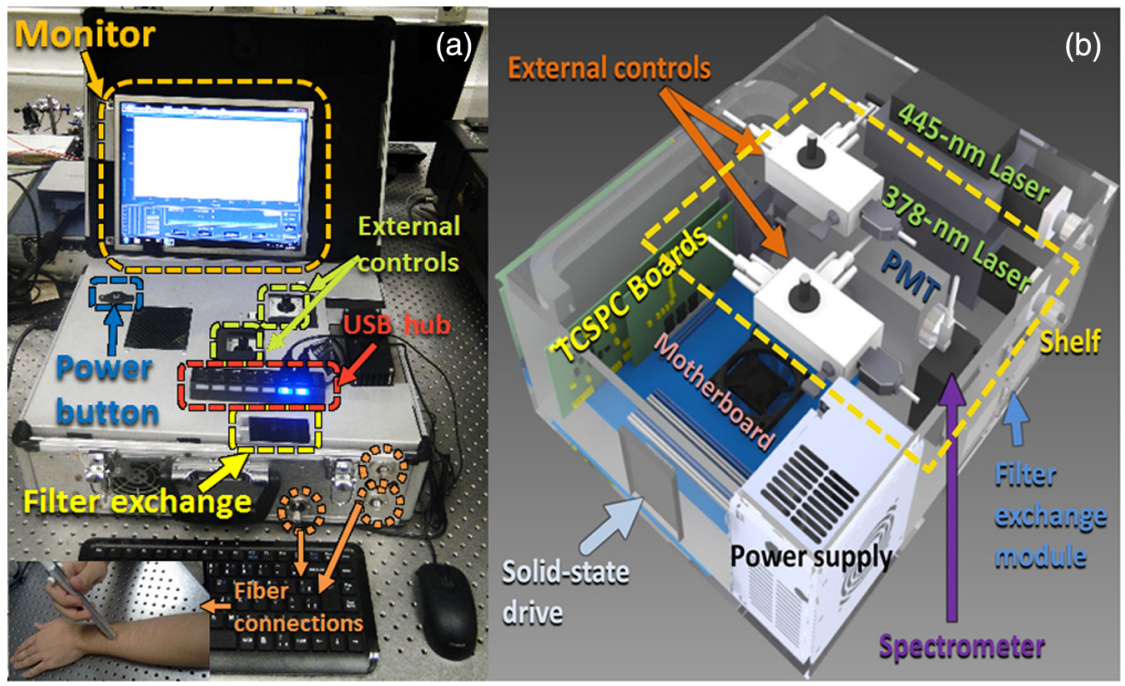

Fig. 3 (a) System assembly after its encasement provides protection for its components and a userfriendly design by exhibiting a control panel with access to external controls and the filter holder. The sample can be placed in any region around the system within the range of the optical fiber ( $2 \mathrm{~m}$ of length). (b) Top view schematic showing the position of each of the system components inside the suitcase. Parts in the upper part of the suitcase, such as 445-nm laser and external controls, are supported by a shelf, which holds the some of the components of the front panel.

using or not using the tip at different conditions: in a dark environment or with illumination from fluorescent lamps, with the fiber at 45-deg surface inclination, and with the fiber optic probe gently touching the tissue surface. Ten measurements were performed for each condition, at the volar forearm of one volunteer, always at the same point and by the same operator.

\subsection{Clinical Measurements}

The subjects were evaluated at the Dermatology Ambulatory of Amaral Carvalho Hospital (Jahu, Sao Paulo, Brazil). The investigated lesions were delimited by the dermatologist and cleaned with a colorless detergent solution (an alcoholic solution of $0.5 \%$ chlorhexidine) without any fluorescence emission under the excitation wavelengths used in this study. Skin lesions measurements were performed at five sites inside each lesion area and five sites at clinically normal tissue in the surrounding area of each lesion (at the same anatomical site), delimited by the dermatologist. The study protocol was approved by the Research Ethics Committee of Amaral Carvalho (38719514.8.0000.5434), and the six enrolled patients signed an informed consent form. To evaluate the clinical feasibility of our system, three hypochromic nevus $(\mathrm{HN})$, three BCCs, and adjacent normal tissue for each of the six abnormal regions were interrogated.

\subsection{Spectral Data Processing}

Each acquired decay profile was processed using the SPCImage software (Becker and Hickl, Berlin, Germany). For the determination of Rhodamine $6 \mathrm{G}$ lifetime, the fluorescence decay curve was fitted by a monoexponential decay for both 378- and 445nm excitations, $20-$ and $50-\mathrm{MHz}$ repetition rates. These repetition rates were evaluated to check the reproducibility of our fluorescence lifetime values when considering the trade-off between the time range and temporal resolution of the acquired fluorescence decay, since we can record only 1024 points in each measurement. For skin lesions measurements, the decay curve was fitted by bi- and triexponential decays. We obtained relative coefficients and lifetimes for these multiexponential decays by performing a fitting of the experimental fluorescence decay $F(t)$ [Eq. (1)] to the convolution between the IRF (about $300 \mathrm{ps}$ of full-width at half-maximum) with a multiexponential decay function

$$
F(t)=\operatorname{IRF} \times \sum a_{i} e^{-\frac{t}{\tau_{i}}}, \quad i=2 \text { or } 3,
$$

where $t$ is the time after the excitation pulse of light, $i$ is the number of exponentials, $a_{i}$ are the relative coefficients of each exponential component, and $\tau_{i}$ are the lifetimes for each component, respectively. Initial analyses were performed by calculating the intensity and amplitude average lifetime [Eqs. (2) and (3)] for normal tissue, and benign and malignant lesions, for each excitation wavelength

Intensity average lifetime $=$ Intensity $\tau_{\mathrm{avg}, n}=\frac{\sum_{1}^{n} a_{i} \tau_{i}^{2}}{\sum_{1}^{n} a_{i} \tau_{i}}$,

Amplitude average lifetime $=$ Amplitude $\tau_{\mathrm{avg}, n}=\frac{\sum_{1}^{n} a_{i} \tau_{i}}{\sum_{1}^{n} a_{i}}$

where $n$ stands for the number of exponential decays taken into account.

The average intensity lifetime is the average amount of time a fluorophore spends in its excited state before returning to the ground state and is calculated by averaging $t$ over the intensity decay, which is proportional to $a \tau \cdot{ }^{10,48}$ On the other hand, the amplitude average lifetime is the lifetime a fluorophore would have if its steady-state fluorescence was the same as a fluorophore or multiple fluorophores with several lifetimes. ${ }^{48-50}$ In biological tissues, even though both average lifetimes provide information about the microenvironment of targeted 
fluorophores in tissue, the first one is independent of the final steady-state intensity, whereas the second one is associated to the optical processes that will result in changes in this intensity. In our case, we expect to extract features from the local microenvironment of the fluorophores with the intensity $\tau_{\text {avg }}$ and information from all the interrogated volume with the amplitude $\tau_{\text {avg }}$.

The average lifetimes of each investigated biological tissue are individually shown on a boxplot graph (Figs. 5 and 6). The statistical differences between the groups were evaluated using a Wilcoxon rank sum test $(p<0.001)$ when comparing HN with $\mathrm{BCC}$, and a Wilcoxon signed-rank test $(p<0.001)$ for comparisons between normal tissue and each skin lesion considering the Bonferroni correction for multiple comparisons.

\section{Results and Discussion}

\subsection{Instrument Response Function and System Calibration}

The IRF was obtained by measurements of the temporal pulse width after the laser pulse passes through excitation and collecting fibers and a little distance (typically 100 to $300 \mu \mathrm{m}$ for the wavelengths used in this study) in a medium with high scatterer concentration, which induces an immediate scattering of the laser pulse and a minimal detection of deeper ballistic photons. The IRF measurements showed that the laser pulse temporal width is 280 ps for the $378-\mathrm{nm}$ laser and 310 ps for the 445nm laser, making it possible to obtain accurate measurements for most of the average lifetimes of molecules present in biological tissues (200 to $5000 \mathrm{ps}$ ). In addition, this temporal width allows a satisfactory fluorescence lifetime resolution to distinguish the shortest lifetime among the fluorophores to be monitored in future biological tissues (NADH and FAD), which can reach lifetime values between 300 and 400 ps.

After determining the IRF, we evaluated the Rhodamine 6G solution by its absorption and fluorescence spectra, which were similar to the ones presented by its manufacturer. ${ }^{8}$ The Rhodamine 6G fluorescence decay was measured using 20and $50-\mathrm{MHz}$ laser repetition rates and both measurements resulted in the average lifetime obtained for Rhodamine. The average value was $(4.1 \pm 0.2) \mathrm{ns}$, which is the same as what is reported in the literature: $4.1 \mathrm{~ns}$ (in water solution). ${ }^{51-54}$ The agreement between these values indicates that our fluorescence lifetime spectroscopy system was properly calibrated.

\subsection{Customized Fiber Tip Improves the Stability of In-Vivo Measurements}

To improve the reproducibility of the clinical measurements in biological tissues, a fiber tip was designed to make the measurements less influenced by distinct clinical environments, especially concerning ambient light and operator handling. The tip operation was evaluated by simulating the conditions where future measurements could be performed in clinics. Measurements of volar forearm tissue with or without the fiber tip showed no difference among steady-state fluorescence spectra normalized by the intensity maximum (data not shown).

For time-resolved measurements, since the increase of the number of exponential components may induce unwanted overfitting of the fluorescence decay, we would like to use as less exponential components as possible and extract the highest amount of biochemical information we can. Then, we calculated average fluorescence lifetimes for bi- and triexponential decays in order to evaluate potential fluctuations due to changes in the composition of the interrogated tissue volume for each environmental condition (angled at $45 \mathrm{deg}$, illuminated, and pressured) and due to the solution obtained from the fitting process. In our measurements, both bi- and triexponential fittings showed the same value for the average lifetime for all the groups, which could indicate that the biexponential fitting was sufficient to describe fluorescence decays (Figs. 4 and 5).

Measurements using the fiber tip showed a higher reproducibility and stability. This stability can be confirmed by lower variations of average fluorescence lifetimes on measurements in a dark environment (Figs. 4 and 5). Furthermore, when the fiber tip was used, we observed fewer changes in both mean values of intensity and amplitude average lifetimes among the different conditions presented in this study (Figs. 4 and 5). The coefficient of variation (CV) was also smaller for most of the cases when using the tip. When the probe is pressed in tissue, almost no difference in the CV was obtained using this tip. Regarding measurements using bandpass filters, the use of the tip allowed more stability compared to the longpass ones, whose use led to very similar average lifetimes in the presence or absence of the tip (data not shown).

\subsection{System Validation: In-Vivo Measurements of Skin Lesions}

The system validation was performed by comparing the fluorescence spectra and lifetimes for normal tissue and two types of skin lesions (HN and BCC). The fluorescence spectra for $378 \mathrm{~nm}$ were very similar for the three tissue types, but, when using the 445-nm excitation, HN and normal tissue had slightly lower intensities compared to BCC in the 520- to 700-nm and 580- to 700-nm spectral regions, respectively (Fig. 6).

To show the data distribution for each interrogated tissue (HN, BCC, and normal tissue), we chose to present them in boxplot graphs of average fluorescence lifetimes (Figs. 7 and 8). In these boxplots, the upper and lower lines indicate the 25th and 75 th percentiles, whereas upper and lower whiskers indicate the 5th and 95th percentiles, respectively. The line in the middle of the box represents the median, and the point in its center is the mean of the data distribution. Based on these boxplots, the fluorescence lifetime values showed a tendency to be lower for BCC when using the 378-nm excitation [Figs. 7(a), 7(b), 8(a), and $8(\mathrm{~b})]$ and for $\mathrm{HN}$ when using the 445-nm excitation [Figs. 7(c), 7(d), 8(c), and 8(d)].

We could discriminate between each combination of clinically similar pair-tissues (HN and BCC, $\mathrm{HN}$ and normal tissue, BCC, and normal tissue) showed in this study. The significant statistical difference [Wilcoxon rank sum test $(p<0.001)$ for comparisons of $\mathrm{HN}$ between BCC and Wilcoxon signed-rank test $(p<0.001)$ for normal tissue and each lesion] is indicated by the star symbol in Figs. 7 and 8. A better differentiation was observed in the average amplitude lifetimes. By combining data from 378- and 445-nm excitations, the discrimination between each pair of tissues in an automated system may be possible. Moreover, this combination can improve the differentiation among tissues when using multivariate analysis techniques.

We believe the main reasons for this differentiation are the concentration of chromophores in tissue, such as melanin and hemoglobin, and changes in the fluorescence of keratin, $\mathrm{NADH}, \mathrm{FAD}$, elastin, and collagen. The first fluorophore is mostly present in the outermost stratum of the epidermis 

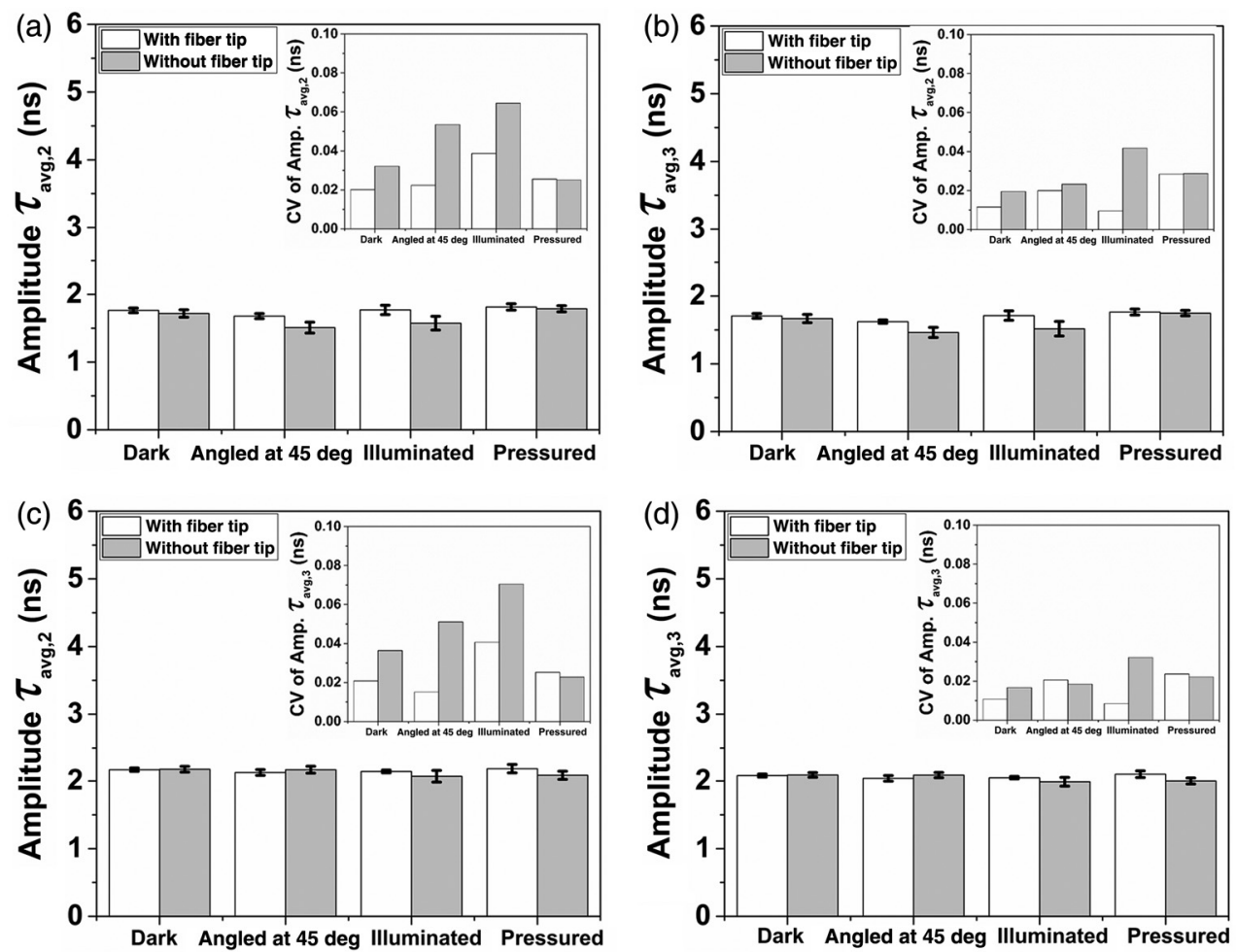

Fig. 4 Amplitude fluorescence lifetimes and their respective CVs when using bandpass filters for (a and b) 378-nm and (c and d) 445-nm excitations. Measurements using the probe were more stable and reproducible.
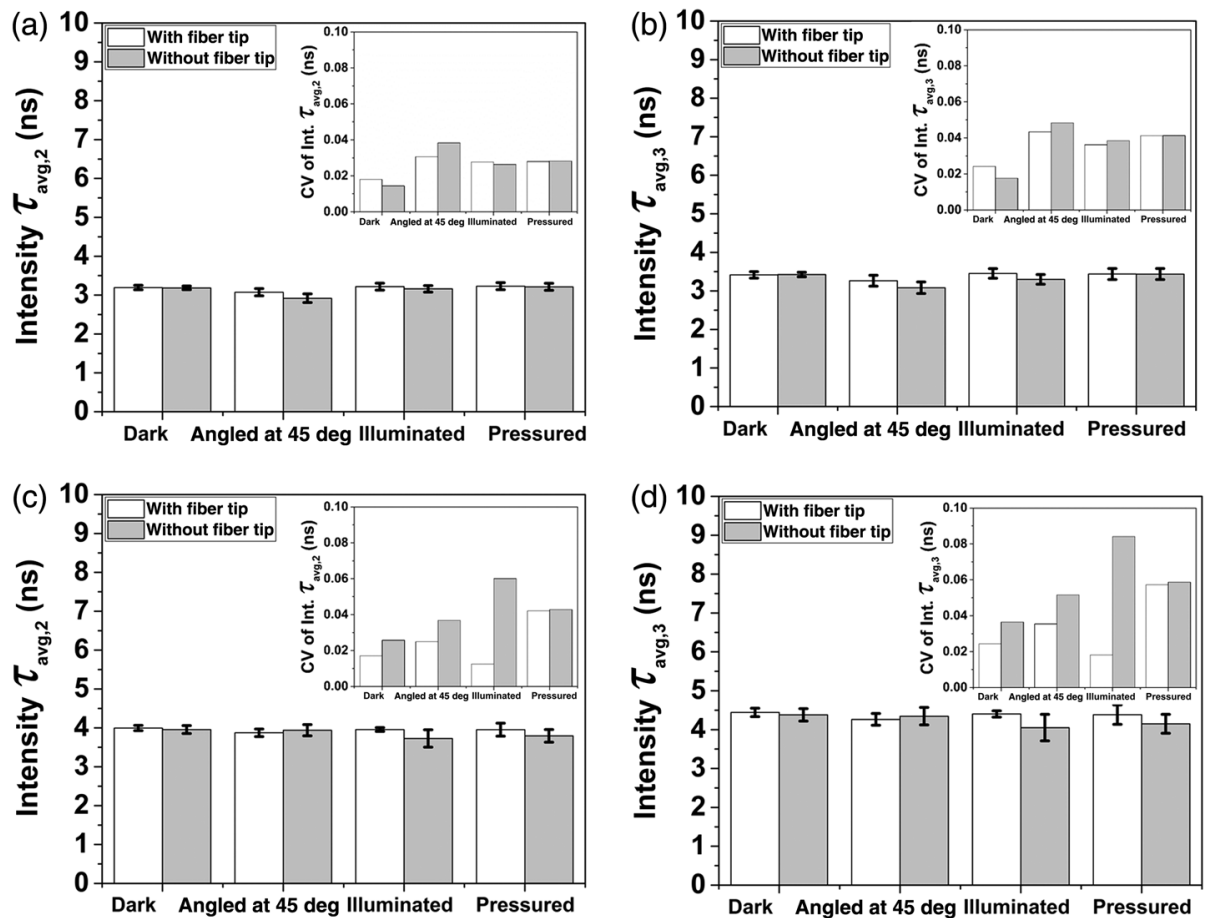

Fig. 5 Intensity fluorescence lifetimes and their respective $C V$ s when using bandpass filters for ( $a$ and $b$ ) $378-\mathrm{nm}$ and (c and d) 445-nm excitations. Measurements using the probe were more stable and reproducible.

(keratinocytes). A higher contribution of the second and third ones arises from cells in part of the dermis and in the lower sections of the epidermis. ${ }^{33}$ Depending on the thickness of the epidermis, we may be able to detect differences of collagen and elastin in the dermis. The dermis has a dense collagen network, which, in BCC lesions, is degraded by tumor-associated matrix metalloproteinases. Then, this degraded collagen is replaced by cell-rich BCC tumor nests. ${ }^{34}$ The tumor cells have a different 

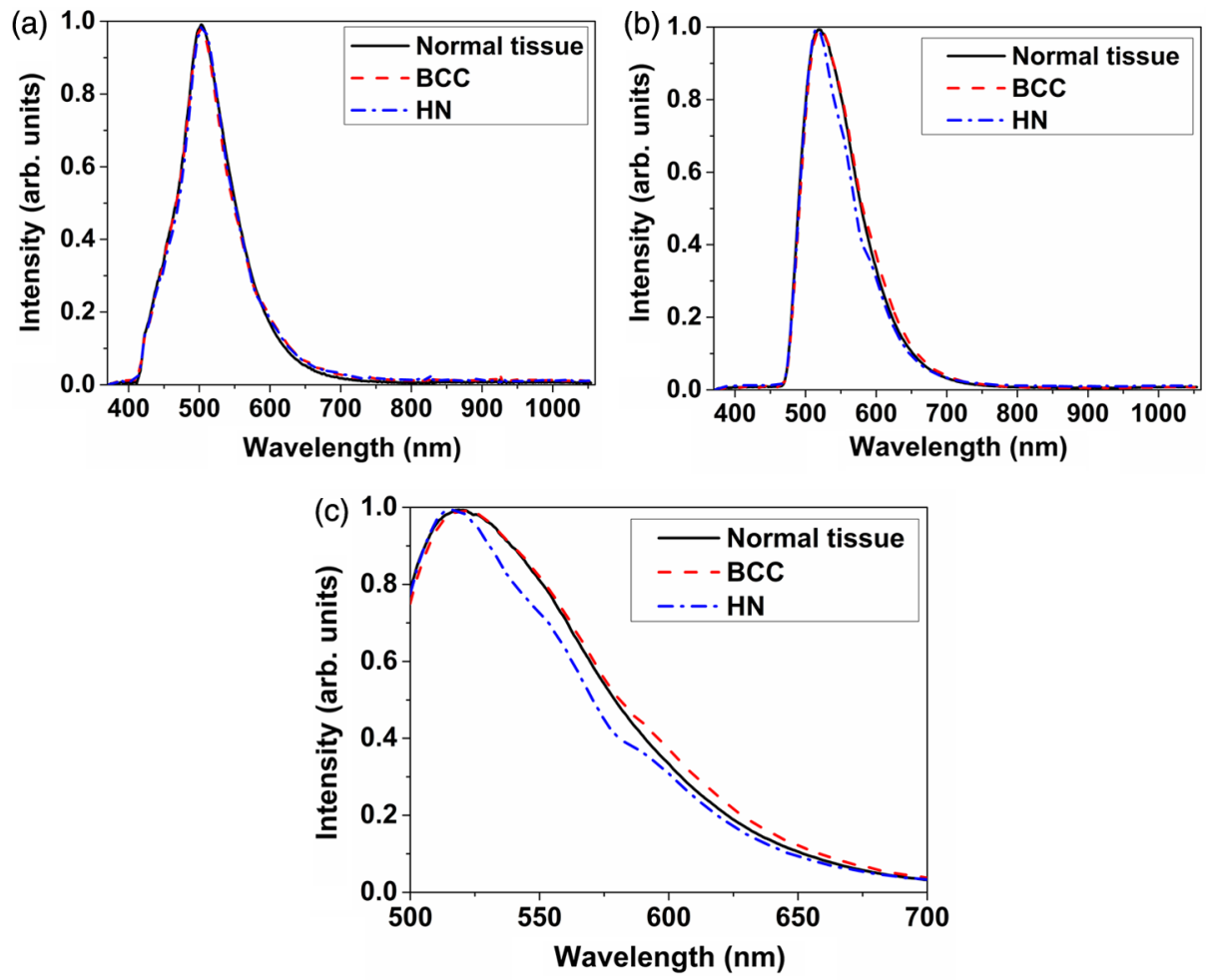

Fig. 6 Average fluorescence spectra of normal tissue, HNs, and BCC for (a) 378-nm and (b, c) 445-nm excitations.
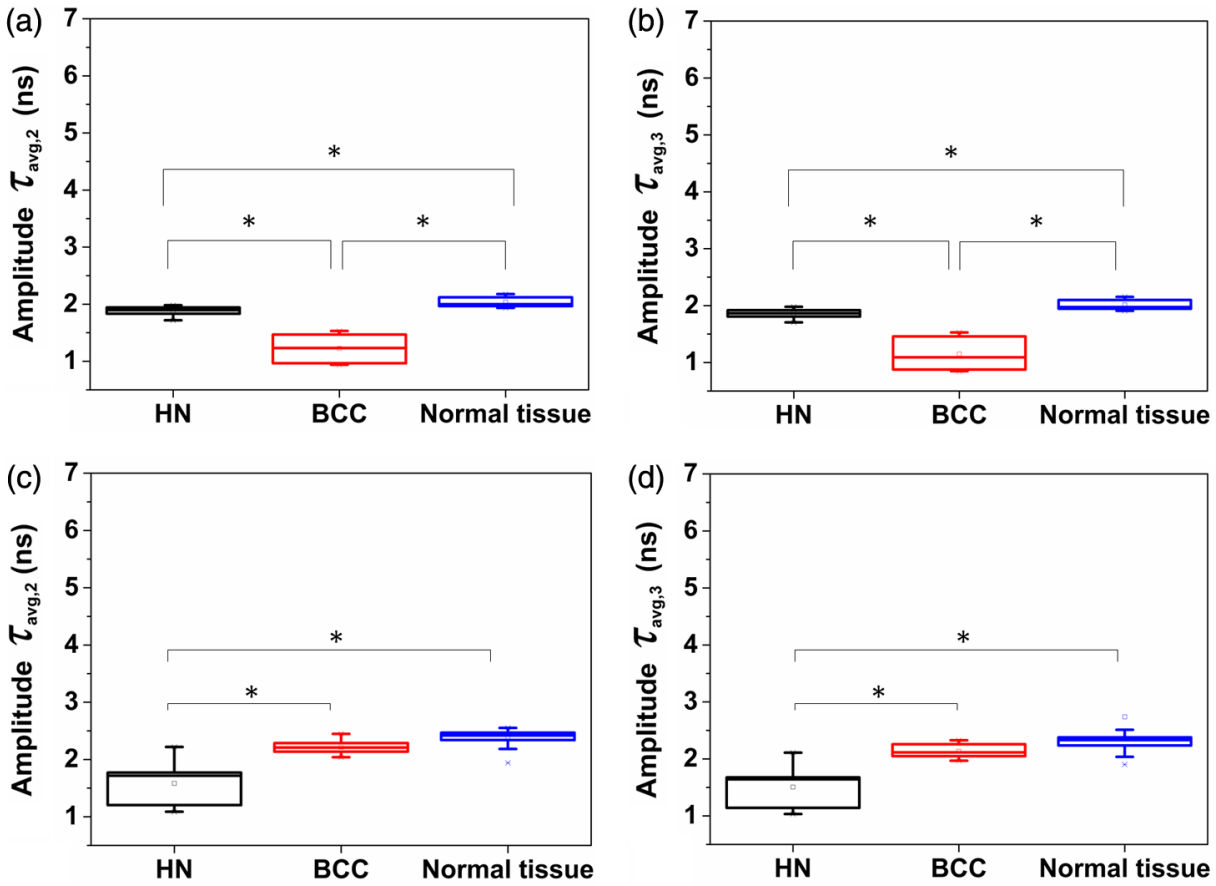

Fig. 7 Boxplot graphs of amplitude average fluorescence lifetimes when using bandpass filters for (a and b) 378-nm and (c and d) 445-nm excitations. For $378 \mathrm{~nm}$, lifetimes were lower for BCC, and for $445 \mathrm{~nm}$, for $\mathrm{HN}$, when compared to the other groups. The amplitude values showed an improved discrimination when compared to the intensity average lifetimes. The $\left(^{*}\right)$ symbol shows each pair with significant statistical difference $(p<0.001$, Wilcoxon rank sum test when comparing $\mathrm{HN}$ with $\mathrm{BCC}$, and $p<0.001$, Wilcoxon signed-rank test for differences between normal tissue and each skin lesion). 

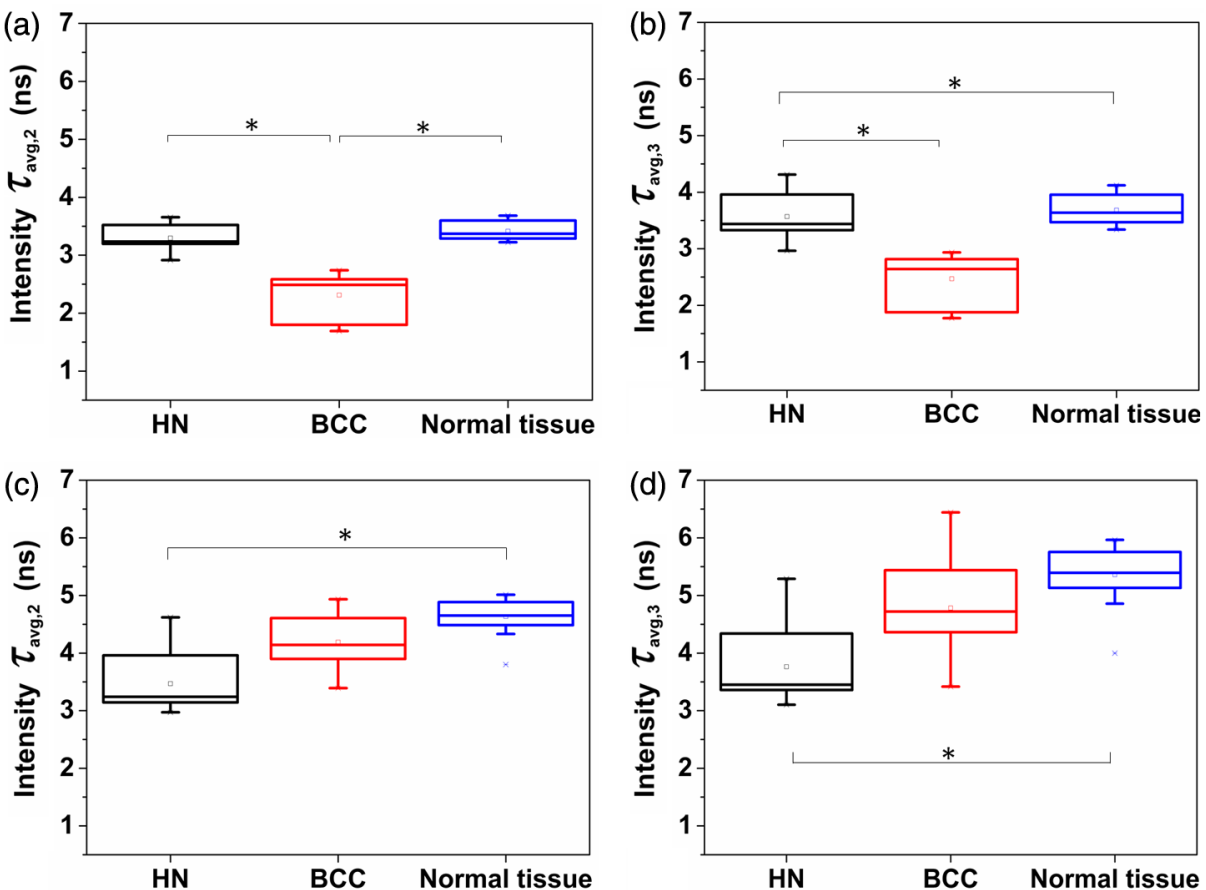

Fig. 8 Boxplot graphs of intensity average fluorescence lifetimes when using bandpass filters for (a and b) 378-nm and (c and d) 445-nm excitations. The * symbol shows each pair with significant statistical difference $(p<0.001$, Wilcoxon rank sum test when comparing $\mathrm{HN}$ with $\mathrm{BCC}$, and $p<0.001$, Wilcoxon signed-rank test for differences between normal tissue and each skin lesion).

metabolic activity due to the Warburg effect, ${ }^{55}$ thus leading to changes in free and bound states of NADH and FAD, and the fluorescence lifetimes we measure. ${ }^{16}$ Also, differences in collagen content and elastin microenvironment may be causes of the changes in the fluorescence lifetime values we obtained. In regards to the contribution of the chromophores, since melanin is a strong absorber for short wavelengths, ${ }^{56}$ it decreases the light penetration of the excitation sources we are using (378 and $445 \mathrm{~nm}$ ). Then, we can collect fluorescence from deeper layers of skin in $\mathrm{HN}$, which has less melanin content than normal tissue and BCC. ${ }^{57}$ Also, due to hemoglobin absorption, increased blood content in BCCs could also attenuate both excitation and fluorescence light. ${ }^{34}$ When using the average amplitude lifetime for 378-nm excitation measurements, it was possible to differentiate all combinations among lesions and normal tissue. This suggests that contributions of fluorescence of collagen, elastin, NADH, and absorption of melanin and hemoglobin are crucial factors for this differentiation. For all cases, a shift to lower values of both amplitude and intensity was observed when comparing normal tissue with BCC. This trend was also observed in other results reported in the literature, ${ }^{30,33,34}$ in agreement with their description. Using the same statistical test as by Thompson et al., ${ }^{30}$ we achieved a $p$-value of $6 \times 10^{-5}$ for analysis of the pair $\mathrm{HN}-$ normal tissue, which is similar to their result of a $5.6 \times 10^{-6} p$-value for the comparison between measurements in situ of normal skin tissue and a set of lesions (BCC, SCC, benign naevus, dysplastic naevus, malignant melanoma, and AK). In addition, we could discriminate among similar lesions, as described by Galletly et al., who observed significant statistical differences $(p<0.05$, Mann-Whitney U-test) in the average fluorescence lifetime when comparing measurements ex situ of nodular and superficial BCCs with infiltrative, micronodular, and mixed subtypes of BCC in an ex-vivo study. ${ }^{34}$ Although these are preliminary data to validate the system, they show the potential of a portable fluorescence lifetimes spectroscopy system for clinical measurements and investigation of biological tissues in vivo.

\section{Conclusions}

In this study, we assembled a portable clinical system for timeresolved fluorescence spectroscopy. This system can be transported with mechanical, electrical, and optical stability, allowing a fast optical filters change during clinical measurements and has $38,166 \mathrm{~cm}^{3}$ of occupied volume.

The system was characterized and calibrated, and the Rhodamine $6 \mathrm{G}$ lifetime value obtained was $4.1 \pm 0.2 \mathrm{~ns}$, in agreement with results reported in the literature. The system can measure average lifetimes of most of the biological tissue molecules (lifetimes between 0.2 and $5 \mathrm{~ns}$ ), including $\mathrm{NADH}$ and FAD.

The system was also validated by performing measurements on different situations/environments and tissues. When using the customized fiber tip, measurements became more stable, and fewer variations for normal tissue were observed. The successful differentiation between each lesion ( $\mathrm{HN}$ and $\mathrm{BCC})$ showed the potential of our fluorescence lifetime spectroscopy system to discriminate these lesions in dubious cases, aiding to the clinical detection and further patient management. Moreover, since normal tissue can be distinguished from the two types of clinically similar skin lesions, our system could be used to identify superficial BCC cells remaining in the surrounding normal tissue after resection as well. These results suggest this system is robust and feasible for clinical measurements of biological tissues. 


\section{Disclosures}

The authors have no relevant financial interests in this article and no potential conflicts of interest to disclose.

\section{Acknowledgments}

The authors acknowledge the support provided by the Brazilian Funding Agencies: Coordination for the Improvement of Higher Education Personnel-CAPES; National Council for Scientific and Technological Development-CNPQ (Universal 307087/ 2013-1 and CK research support); and São Paulo Research Foundation-FAPESP [Grants Nos. 2013/07276-1 (Optics and Photonics Research Center-CePOF), 2014/16154-0 (MSN scholarship), and 2014/50857-8 (National Institute of Science and Technology-INCT)].

\section{References}

1. N. Ramanujam, "Fluorescence spectroscopy in vivo," in Encyclopedia of Analytical Chemistry, John Wiley \& Sons, Ltd., Chichester (2006).

2. G. A. Wagnieres, W. M. Star, and B. C. Wilson, "In vivo fluorescence spectroscopy and imaging for oncological applications," Photochem. Photobiol. 68(5), 603-632 (1998).

3. N. Ramanujam, "Fluorescence spectroscopy of neoplastic and non-neoplastic tissues," Neoplasia 2(1-2), 89-117 (2000).

4. M. Y. Berezin and S. Achilefu, "Fluorescence lifetime measurements and biological imaging," Chem. Rev. 110(5), 2641-2684 (2010).

5. M. C. Skala et al., "In vivo multiphoton microscopy of NADH and FAD redox states, fluorescence lifetimes, and cellular morphology in precancerous epithelia," Proc. Natl. Acad. Sci. U. S. A. 104(49), 19494-19499 (2007).

6. C. de Paula Campos et al., "Fluorescence spectroscopy in the visible range for the assessment of UVB radiation effects in hairless mice skin," Photodiagn. Photodyn. Ther. 20, 21-27 (2017).

7. M. Saito Nogueira et al., "Evaluation of actinic cheilitis using fluorescence lifetime spectroscopy," Proc. SPIE 9703, 97031U (2016).

8. M. Saito Nogueira et al., "Assembly and characterization of a fluorescence lifetime spectroscopy system for skin lesions diagnostic," Proc. SPIE 9531, 95313D (2015).

9. C. de Paula D'Almeida et al., "Time-resolved and steady-state fluorescence spectroscopy for the assessment of skin photoaging process," Proc. SPIE 9531, 953146 (2015).

10. J. R. Lakowicz, Principles of Fluorescence Spectroscopy, 3rd ed., Springer, Baltimore (2006).

11. L. Marcu, P. French, and D. Elson, Fluorescence Lifetime Spectroscopy and Imaging, L. Marcu, P. French, and D. Elson, Eds., CRC Press, Boca Raton, Florida (2014).

12. E. Gratton et al., "Fluorescence lifetime imaging for the two-photon microscope: time-domain and frequency-domain methods," $J$. Biomed. Opt. 8(3), 381 (2003).

13. W. Becker, Advanced Time-Correlated Single Photon Counting Techniques, A. W. Castleman, J. P. Toennies, and W. Zinth, Eds., Springer Berlin Heidelberg, Berlin, Heidelberg (2005).

14. M. C. Skala et al., "In vivo multiphoton fluorescence lifetime imaging of protein-bound and free nicotinamide adenine dinucleotide in normal and precancerous epithelia," J. Biomed. Opt. 12(2), 024014 (2007).

15. A. Cosci et al., "Time-resolved fluorescence spectroscopy for clinical diagnosis of actinic cheilitis," Biomed. Opt. Express 7(10), 4210 (2016).

16. L. Pires et al., "Time-resolved fluorescence lifetime for cutaneous melanoma detection," Biomed. Opt. Express 5(9), 3080 (2014).

17. K. Koenig and I. Riemann, "High-resolution multiphoton tomography of human skin with subcellular spatial resolution and picosecond time resolution," J. Biomed. Opt. 8(3), 432 (2003).

18. K. Dowling et al., "Fluorescence lifetime imaging with picosecond resolution for biomedical applications," Opt. Lett. 23(10), 810 (1998).

19. D. Elson et al., "Time-domain fluorescence lifetime imaging applied to biological tissue," Photochem. Photobiol. Sci. 3(8), 795 (2004).

20. S. Shrestha et al., "High-speed multispectral fluorescence lifetime imaging implementation for in vivo applications," Opt. Lett. 35(15), 2558 (2010).
21. F. Fereidouni, K. Reitsma, and H. C. Gerritsen, "High speed multispectral fluorescence lifetime imaging," Opt. Express 21(10), 11769 (2013).

22. J. Park et al., "A dual-modality optical coherence tomography and fluorescence lifetime imaging microscopy system for simultaneous morphological and biochemical tissue characterization," Biomed. Opt. Express 1(1), 186-200 (2010).

23. R. Cicchi et al., "Nonlinear laser imaging of skin lesions," $J$. Biophotonics 1(1), 62-73 (2008).

24. H. Sparks et al., "A flexible wide-field FLIM endoscope utilising blue excitation light for label-free contrast of tissue," J. Biophotonics 8(1-2), 168-178 (2015).

25. Y. Sun et al., "Dynamic tissue analysis using time- and wavelengthresolved fluorescence spectroscopy for atherosclerosis diagnosis," Opt. Express 19(5), 3890 (2011).

26. J. M. Jabbour et al., "Fluorescence lifetime imaging and reflectance confocal microscopy for multiscale imaging of oral precancer," J. Biomed. Opt. 18(4), 046012 (2013).

27. J. Park et al., "Biochemical characterization of atherosclerotic plaques by endogenous multispectral fluorescence lifetime imaging microscopy," Atherosclerosis 220(2), 394-401 (2012).

28. R. Cicchi et al., "Time- and spectral-resolved two-photon imaging of healthy bladder mucosa and carcinoma in situ," Opt. Express 18(4), 3840 (2010).

29. M. Popenda et al., "Fluorescence lifetime measurements with all-fiber optical setup for non-invasive in-vivo diagnostics," in 17th Int. Conf. on Transparent Optical Networks (ICTON), pp. 1-4 (2015).

30. A. J. Thompson et al., "In vivo measurements of diffuse reflectance and time-resolved autofluorescence emission spectra of basal cell carcinomas," J. Biophotonics 5(3), 240-254 (2012).

31. A. Thompson et al., "Hyperspectral fluorescence lifetime fibre probe spectroscopy for use in the study and diagnosis of osteoarthritis and skin cancer," Proc. SPIE 7895, 78950G (2011).

32. A. J. Thompson, "Developing endoscopic instrumentation and techniques for in vivo fluorescence lifetime imaging and spectroscopy," PhD Thesis, Imperial College London (2013).

33. P. A. A. De Beule et al., "A hyperspectral fluorescence lifetime probe for skin cancer diagnosis," Rev. Sci. Instrum. 78(12), 123101 (2007).

34. N. P. Galletly et al., "Fluorescence lifetime imaging distinguishes basal cell carcinoma from surrounding uninvolved skin," $\mathrm{Br}$. J. Dermatol. 159(1), 152-161 (2008).

35. R. Patalay et al., "Fluorescence lifetime imaging of skin cancer," Proc. SPIE 7883, 78830A (2011).

36. S. Cheng et al., "Flexible endoscope for continuous in vivo multispectral fluorescence lifetime imaging," Opt. Lett. 38(9), 1515 (2013).

37. R. Patalay, "The clinical application of multispectral fluorescence lifetime imaging of human skin using multiphoton microscopy," $\mathrm{PhD}$ Thesis, Imperial College London (2013).

38. Q. Fang et al., "Time-domain laser-induced fluorescence spectroscopy apparatus for clinical diagnostics," Rev. Sci. Instrum. 75(1), 151-162 (2004).

39. P. V. Butte et al., "Fluorescence lifetime spectroscopy for guided therapy of brain tumors," NeuroImage 54(Suppl. 1), S125-S135 (2011).

40. D. Chorvat, Jr. et al., "Multi-wavelength fluorescence lifetime spectroscopy: a new approach to the study of endogenous fluorescence in living cells and tissues," Laser Phys. Lett. 6(3), 175-193 (2009).

41. S. Coda et al., "Fluorescence lifetime spectroscopy of tissue autofluorescence in normal and diseased colon measured ex vivo using a fiberoptic probe," Biomed. Opt. Express 5(2), 515-538 (2014).

42. R. J. Nordstrom et al., "Identification of cervical intraepithelial neoplasia (CIN) using UV-excited fluorescence and diffuse-reflectance tissue spectroscopy," Lasers Surg. Med. 29(2), 118-127 (2001).

43. M. Panjehpour et al., "Laser-induced fluorescence spectroscopy for in vivo diagnosis of non-melanoma skin cancers," Lasers Surg. Med. 31(5), 367-373 (2002).

44. H. Wang et al., "A portable time-domain LED fluorimeter for nanosecond fluorescence lifetime measurements," Rev. Sci. Instrum. 85(5), 55003 (2014).

45. J. Kissinger and D. Wilson, "Portable fluorescence lifetime detection for chlorophyll analysis in marine environments," IEEE Sens. J. 11(2), 288-295 (2011). 
46. J. Enderlein and I. Gregor, "Using fluorescence lifetime for discriminating detector afterpulsing in fluorescence-correlation spectroscopy," Rev. Sci. Instrum. 76(3), 033102 (2005).

47. K. Ishii and T. Tahara, "Correction of the afterpulsing effect in fluorescence correlation spectroscopy using time symmetry analysis," Opt. Express 23(25), 32387 (2015).

48. A. Sillen and Y. Engelborghs, "The correct use of 'average' fluorescence parameters," Photochem. Photobiol. 67(5), 475-486 (1998).

49. S. E. Webber, "The role of time-dependent measurements in elucidating static versus dynamic quenching processes," Photochem. Photobiol. 65(1), 33-38 (1997).

50. R. D. Stramel et al., "Photophysical properties of pyrene covalently bound to photoelectrolytes," J. Chem. Phys. 92(10), 2934-2938 (1988).

51. Q. S. Hanley et al., "Fluorescence lifetime imaging: multi-point calibration, minimum resolvable differences, and artifact suppression," Cytometry 43(4), 248-260 (2001).

52. J. M. Harris and F. E. Lytle, "Measurement of subnanosecond fluorescence decays by sampled single-photon detection," Rev. Sci. Instrum. 48(11), 1469-1476 (1977).
53. D. Magde, G. E. Rojas, and P. G. Seybold, "Solvent dependence of the fluorescence lifetimes of xanthene dyes," Photochem. Photobiol. 70(5), 737-744 (1999).

54. J. R. Lakowicz and K. W. Berndt, "Lifetime-selective fluorescence imaging using an rf phase-sensitive camera," Rev. Sci. Instrum. 62(7), 1727-1734 (1991)

55. I. N. Druzhkova et al., "The metabolic interaction of cancer cells and fibroblasts-coupling between $\mathrm{NAD}(\mathrm{P}) \mathrm{H}$ and $\mathrm{FAD}$, intracellular $\mathrm{pH}$ and hydrogen peroxide," Cell Cycle 15(9), 1257-1266 (2016).

56. G. Zonios et al., "Melanin absorption spectroscopy: new method for noninvasive skin investigation and melanoma detection," J. Biomed. Opt. 13(1), 014017 (2008).

57. H. S. Lee, Y. S. Chun, and S. K. Hann, "Nevus depigmentosus: clinical features and histopathologic characteristics in 67 patients," J. Am. Acad. Dermatol. 40(1), 21-26 (1999).

Biographies for the authors are not available. 